\title{
Protocol of Surgical Treatment of Esophageal Tumor that Depends on Radiotherapy and Chemotherapy
}

\section{Haris Tanović ${ }^{1}$, Edina Tanović ${ }^{2 *}$ and Jusuf Šabanović ${ }^{1}$}

${ }^{1}$ Clinic for Abdominal Surgery, Clinical Center University of Sarajevo, Sarajevo, Bosnia and Hercegovina ${ }^{2}$ Clinic for Physical Medicine and Rehabilitation, Clinical Center University of Sarajevo, Sarajevo, Bosnia and Hercegovina *Corresponding Author: Edina Tanović, Clinic for Physical Medicine and Rehabilitation, Clinical Center University of Sarajevo, Sarajevo, Bosnia and Hercegovina.

Received: September 23, 2019; Published: October 05, 2019

DOI: $10.31080 /$ ASMS.2019.03.0430

\begin{abstract}
Malignant esophageal tumors are one of the most difficult diseases, because when they are noticed, they are already in advanced stage. Sometimes even radical surgical methods can not give desireable results. Results of surgical treatment are best in countries where screening program is included in routine diagnostics. Malignant esophageal tumor takes fourth place out of all tumors of digestive tract, and average age of diseased are between 55. - 65. years. First aim of this study is to analize localization of esophageal tumors, pathohistological representation, operating techniques and complications. Second aim of this study is to show relation between surgical treatment and preoperative radiotherapy and chemotherapy. This retrospective study included 13 patients with pathohistology of esophageal tumor who were on Clinic for abdominal surgery KCUS during two years. All patients had different surgical treatments which depended on stage which was find during surgery. Patients were analyzed based on age, sex, localization of esophageal tumor, and stage of tumor. Analysis of 13 patients included resectional methods, pathohistology and preoperative oncological treatment. Results were different and were corelated with neoadjuvant oncological treatment. Radical surgery had 11 patients that included esophagectomy and esophagoplasty with removal of regional lymphonodes. Esophagogastroplasty was tretment for 6 patients. Becuase of inadequate nutrition or inoperable tumors 6 patients had gastrostomy. Implatation of Haering dentures was included as palliative surgery in 2 patinets. The most of esophageal tumors were located in the lower third (53,84\%), and the leaset of esophageal tumors were located in the upper third (15,83\%). Pathohistology analysis of esophageal tumors showed that tumors were planocellular carcinoma (67\%) and adenocarcinoma (23\%). In most cases radical surgery treatment was required. Tumors of lower third esophagus are most numerous, and least numerous are in upper third. Planocellular carcinoma is the most often malignant tumor of esophagus. Radical surgical approach was used 84,6\% patients and the rest patients $15,4 \%$ had palliative surgery. The most used techniques was gastroplasty which was the best and from palliative sugery was gastrostomy. Postoperative complications were present in $23,1 \%$ of cases. Reactions of tumor on chemotherapy and radiotherapy are different, sometimes even remission of malignant cell is possible. Even in that cases radical surgical approach remains the best method in every case after chemotherapy and radiotherapy.

Keywords: Esophageal Tumors; Radiotherapy; Chemotherapy
\end{abstract}

\section{Abbreviations}

CT scan: Computed Tomography; EUS: Endoscopic Ultrasound; NCCN: National Comprehensive Cancer Network; PET: Positron Emission Tomography; TRG: Tumor Regression Grade

\section{Introduction}

Surgical division of esophagus is on the neck part (pars cevicalis) which starts from the beginning of esophagus $(15 \mathrm{~cm})$, together with upper thoracal part and ends at notch in manubrium of sternum $(24 \mathrm{~cm})$. After that part, comes the chest part of oesophagei (pars thoracalis medialis) that is connected to previous $24 \mathrm{~cm}$, where starts medium thoracal part and goes threw the port on diaphragm (hiatus oesophagei), and final part ends with ostium cardiacum $(38 \mathrm{~cm})[1]$.

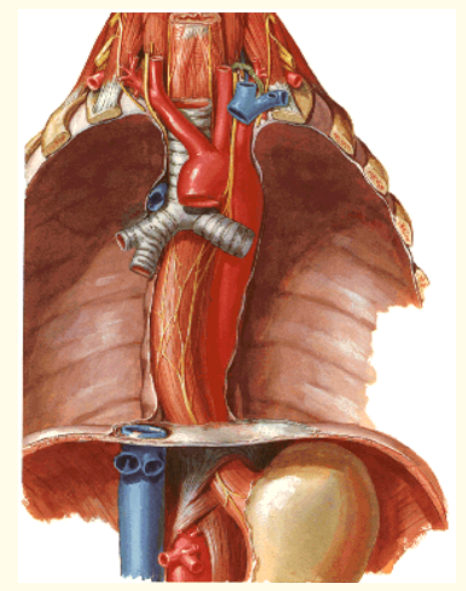




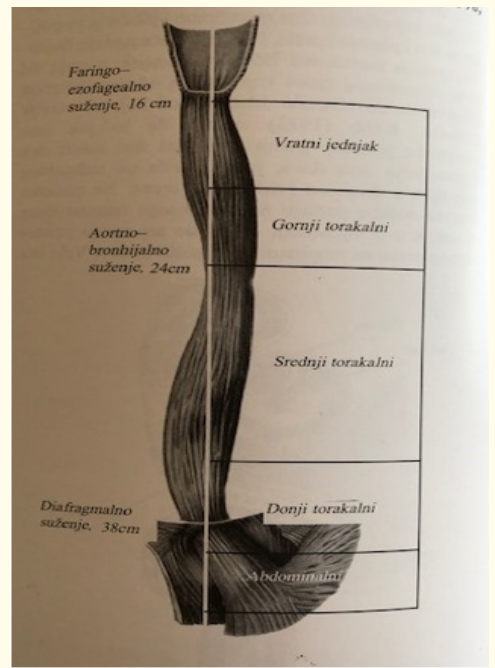

Figure 1 and 2: Anatomy of esophagus [1].

Malignant esophageal tumors are one of the most difficult diseases, because when they are noticed, they are already in advanced stage. Sometimes even radical surgical treatment can not give desirable results. Primary esophageal tumors can be malignant esophageal tumors of epidermal etiology $>90 \%$. These carcinoms can be squamocellular (70 - 80\%) and adenocarcinoma (10 - 30\%). Sometimes, they are made from Leiomiosarcoma and Rabdomyomas of mesodermal etiology (less than 1\%). From benign esophageal tumors most are made from planocellular papillomas that have epidermal etiology, or Leiomyoma and fibrovascular papilloma that have mesodermal etiology. Secondary esophageal tumors are very rare [2].

Results of radical surgical approach are not promising, because they usually have bad outcomes, and they are the best in countries with screening program included in routine. Malignant esophageal tumor takes fourth place out of all tumors of digestive tract, and average age of diseased is between 55. - 65. years [3].

Normal esophagus has most active peristalsis in digestive tract [4].

Reconstruction in surgery includes regeneration of established continuity, ability to pass, appearance and composition, that leads to normal function which is the most important thing [1]

The basic principle of surgical technique is that part of esophagus can be replaced only with vital parts of digestive tube, that has digestive activity, and ability to pass as the most important thing [5].

Different parts are available as replacement, and each of them has its own advantages and disadvantages, because different parts have different pathophysiological characteristics [4].
Jejunum has the best peristalsis, colon has the best vascularization, and the stomach has the best functionality and has least outcomes with complications [1]

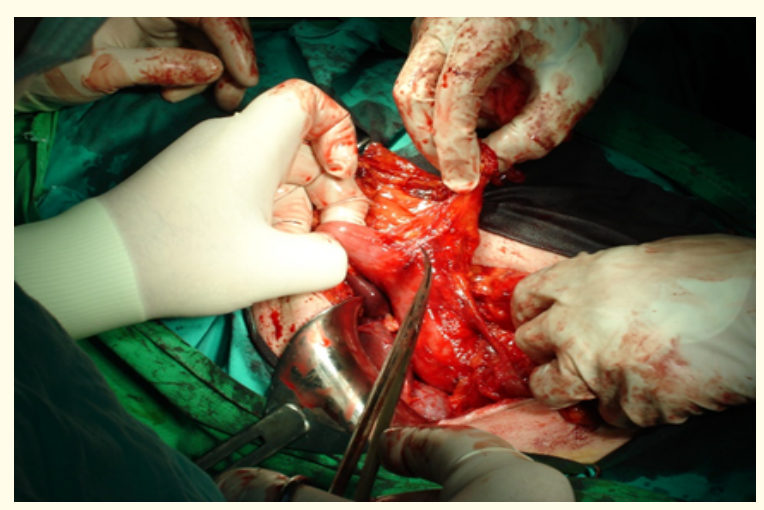

Figure 3: Preparation digestive tube for transposition
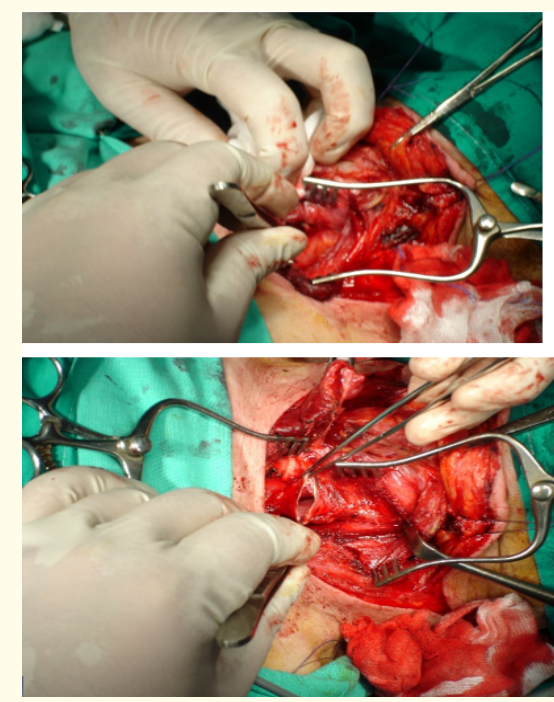

Figure 4 and 5: Preparation of neck part of esophagus.

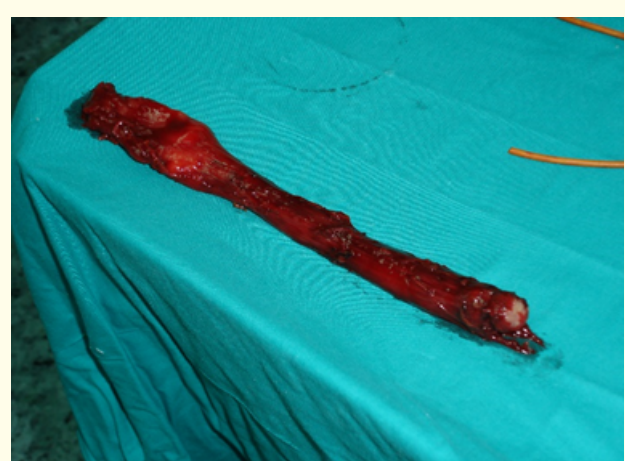

Figure 6: Resected esophageal tumor. 

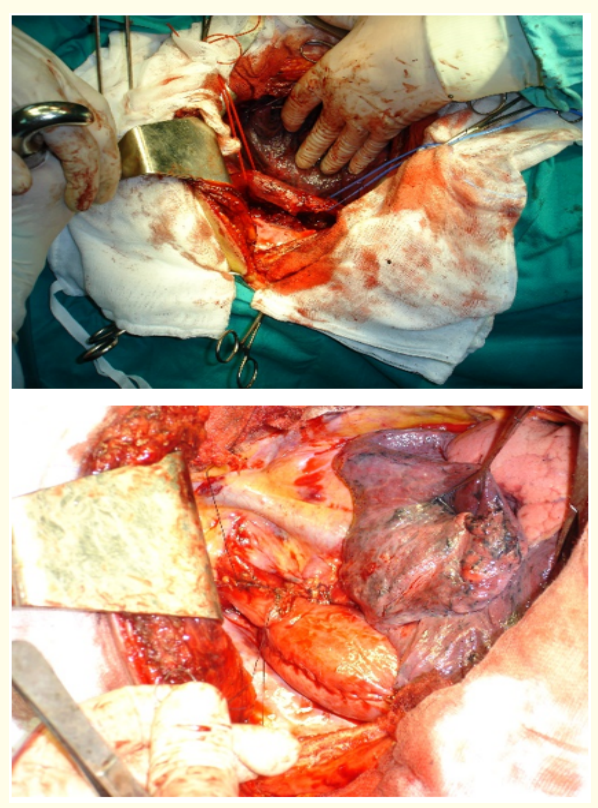

Figure 7 and 8: Preparation of chest part of esophageal tumor and anastomosis with stomach after resection of tumor.

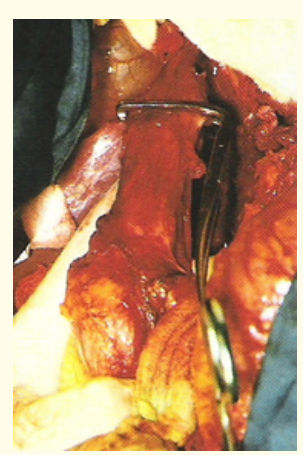

Figure 9: Preparation for resection of lower part in esophagus.

Palliative surgery are used, sometimes if resection is not possible, and they are operated with „By pass“, bridging stenosis, different prosthesis or gastric stoma. Today gastric stoma is chosed as method, because it has the least outcomes with complications [6].

First aim of this study is to analize localization of esophageal tumors, pathohistological representation, operating techniques and complications. Second aim of this study is to show relation between surgical treatment and preoperative radiotherapy and chemotherapy.

\section{Materials and Methods}

This retrospective study included 13 patients with pathohistology of esophageal tumor who were on Clinic for abdominal surgery KCUS during two years. Surgical approaches were analyzed based on preoperative radiotherapy and chemotherapy. All patients had different surgical treatments which depended on stage which was find during surgery. Patients were analyzed based on age, sex, localization of esophageal tumor, and stage of tumor. Esophageal tumors were observed together with surgical approaches, pathohistological representation and possible complications. It was important to establish connection between resectional methods and pathohistological representation and preoperative oncological treatment.

Statistical analysis was made using Hi-square test, where results of this tests showed significant results in reability of $95 \%$ or $\mathrm{p}<0,05$.

\section{Results}

Results were shown in tabels and graphs with number of cases, percentage, distribution of gender.

\begin{tabular}{|l|c|c|c|}
\hline & Total & Male & Female \\
\hline Number of Patients & 13 & 9 & 4 \\
\hline Percentage & $100 \%$ & $69,2 \%$ & $30,8 \%$ \\
\hline
\end{tabular}

Table 1: Gender distribution.

Gender distribution shows that in this group was more man with 9 or $69,2 \%$ compared to woman with 4 or $30,8 \%$. Statistical analysis form Hi-square test shows that there is no significant difference between gender compared to expected $(p>0,05)$.

\begin{tabular}{|l|c|c|c|}
\hline \multicolumn{1}{|c|}{ Age } & $\mathbf{0 - 4 5}$ & $\mathbf{4 5 - 6 5}$ & $>\mathbf{6 5}$ \\
\hline Number of Patients & 1 & 6 & 6 \\
\hline Percentage & 7,7 & 46,2 & 46,2 \\
\hline
\end{tabular}

Table 2: Age structure.

Statistical analysis shows that the least of patients were at age range $0-45$, or $7,7 \%$ of patients. The majority of patients were at age range above 45 or $92.4 \%$, from which $46,2 \%$ were at age range $45-65$, and other $46,2 \%$ were at age range above 65 years.

On graph 1. localization of tumor is shown where tumors were located: in the lower third was 53,84\%, middle third $23.07 \%$, upper third $15,38 \%$ and undefined was 7,69\%. In patients, 7 of them had esophageal tumor in lower third, 3 of them had esophageal tumor in middle third, 2 of them had esophageal tumor in upper third, 1 of them had undefined localization of esophageal tumor.

Patohistological analyze showed that planocellular carcinoma had 9 patients, following with adenocarcinoma that had 3 patients, and 1 patient was undefined. Statistical analyze showed that most of patients $67 \%$ had planocellular carcinoma, and $23 \%$ of patients had adenocarcinoma, while $11 \%$ was undefined.

Statistical analyze showed that most of the patients $84,6 \%$ had radical surgery approach, while $15,4 \%$ of patients had palliative surgery. 


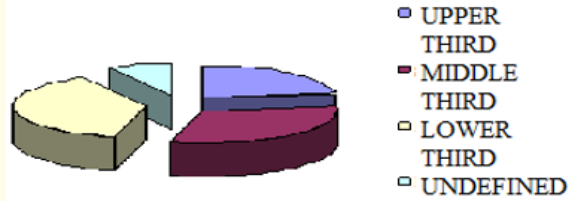

Graph 1: Localization of esophageal tumors.
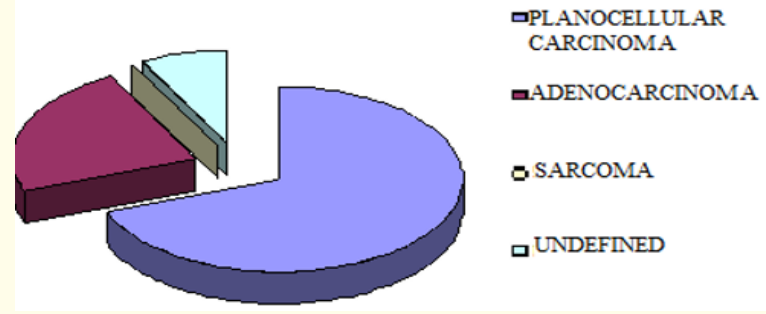

Graph 2: Type of tumor.

\begin{tabular}{|l|c|c|}
\hline \multicolumn{1}{|c|}{ Type of Surgery } & Radical & Palliative \\
\hline Number of Patients & 11 & 2 \\
\hline Percentage & 84,6 & 15,4 \\
\hline
\end{tabular}

Table 3: Type of surgical approach.

On graph 3. complications that may occur are shown, where 3 patients had complications or $23,1 \%$, and other patients 10 of them had not complications or $76,9 \%$.
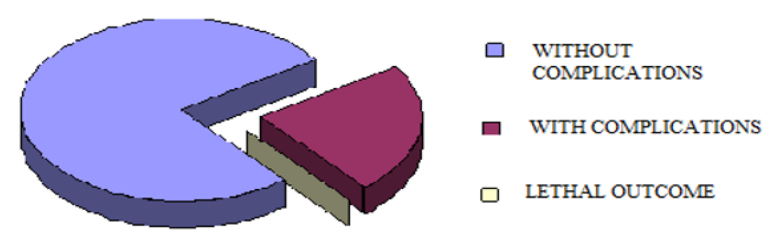

Graph 3: Complications

\section{Discussion}

Esophageal tumors remain consist challenge in therapy approach. Until now, definitive protocol of treatments is not established yet for the esophageal tumors. The main reason for that is progression in surgical techniques and instruments that are used, as well as inovations of oncological treatments of esophageal tumors. In our research, 13 patients were treated in period of two years.
Radical surgery had 11 patients that included esophagectomy and esophagoplasty with removal of regional lymphonodes. Esophagogastroplasty was tretment for 6 patients. Becuase of inadequate nutrition or inoperable tumors 6 patients had gastrostomy by Witzel [7].

Implatation of Haering dentures was included as palliative surgery in 2 patinets. Two patients had esophagogastric resection, which includes resection on one part of esophagus and one part of stomach, and after that those two parts are connected to make esophagus.

In out research most of esophageal tumors were located in the lower third $(53,84 \%)$, and the leaset of esophageal tumors were located in the upper third $(15,83 \%)$. Pathohistology analysis of esophageal tumors showed that tumors were planocellular carcinoma (67\%) and adenocarcinoma (23\%). In most cases radical surgery treatment was required. Tumors of lower third esophagus are most numerous, and least numerous are in upper third. Planocellular carcinoma is the most often malignant tumor of esophagus. Radical surgical approach was used $84,6 \%$ patients and the rest patients $15,4 \%$ had palliative surgery. The most used techniques was gastroplasty which was the best and from palliative sugery was gastrostomy. Postoperative complications were present in $23,1 \%$ of cases.

Opinion of oncological consilium is very important in choosing of adequate approach. Accurate evaluation of neoadjuvant therapy is challenge in treament of esophageal tumors. Morphology of esophageal tumor is irregular and unequal, meaning that regression of tumor is noticed after treatmen. Tumor regression grade (TRG) is percentage of residual cells of cancer, which is used to define efficiency of neoadjuvant therapy for esophageal carcinoma. First time TRG was proposed on National Comprehensive Cancer Network (NCCN) which was Second Guidline for treatment of esophageal tumors in 2012 [8].

Wu., et al. used TRG as a predictor of patients outcome, because TRG helped them to find focal point of tumor [9]. There is no standard treatmen for local advanced esophageal carcinoma, because treatmen is individual for every single patient. Neoadjuvant therapy or chemoradiotherapy is necessary in treatmen of esophageal carcinoma [10]. Some authors suggests that chemoradiotherapy causes more adverse effects, increased surgical risk, prolonged duration of treatment and bigger financial burdain comparing to chemotherapy itself $[11,12]$.

After neoadjuvant chemotherapy, potential resectable cases of locally advanced cancer are developed in totally respectable cases. Some studies showed that complete pathological response was in less than $20 \%$ of cases after neoadjuvant chemoradiotherapy $[13,14]$. 
Response on therapy is estimated by experienced radiologist, that measured maximal length of tumors and maximal thickness of walls before and after treatment. Results of endoscopy, EUS (endoscopic ultrasound) and CT scans, together with clinical work are discussed by researchers and answer is defined with consensus. Results in PET study are not known for clinical researchers in the time of evaluation of clinical response. Surgical therapy is made of transthoracohiatus block esophagectomy with double lymphadenectomy in tumors that are located around tracheal bifurcations with available jejunal graft and patients with cervical tumor. Surgery remains therapy of choice, if all macroscopic and microscopic resection is available and can be achieved (R0 resection) $[15,16]$.

Preoperative chemotherapy and combined radiotherapy was introduced with primary aim to increase graduate of complete resection with decrease of primary tumor. Control of local tumor and prevention of distance metastases is improved $[17,18]$.

All pathohistological specimens are classified by two experienced pathologists, without other clinical and PET information based on Protocol of International Union against cancer [19].

In valorization of diagnosis $\mathrm{R}$ classification is included. Response on treatment of radiotherapy and chemotherapy is classified as total, subtotal, partial and minimal remission based on criteria made by Mandard., et al [20].

Complete response on radiotherapy and chemotherapy, shows histological fibrosis with or without inflammation that extends to different layers of esophageal wall, but it does not include living residual cells of tumor. Subtotal response is categorized with accessibility under $10 \%$ of visible residual tumor cells, partial response was from $10 \%$ to $50 \%$, and minimal remission more than $50 \%$ of visible residual tumor cells. If there is no change that is defined by absence of regressive change.

Based on results and earlier experience with cells of esophageal carcinoma, it is suggested that next protocol should be used after neoadjuvant treatment. Patients who had answer on neoadjuvant treatment should be subjected to resection of tumor. Patients that are under this category should have surgery if it is possible. For patients that do not have response and have bad prognosis (average os surviving 9 months) it is questionable will they have benefits from surgical resection $[8,20]$.

\section{Conclusions}

Tumors of lower third esophagus are most numerous, and least numerous are in upper third. Planocellular carcinoma is the most often malignant tumor of esophagus.

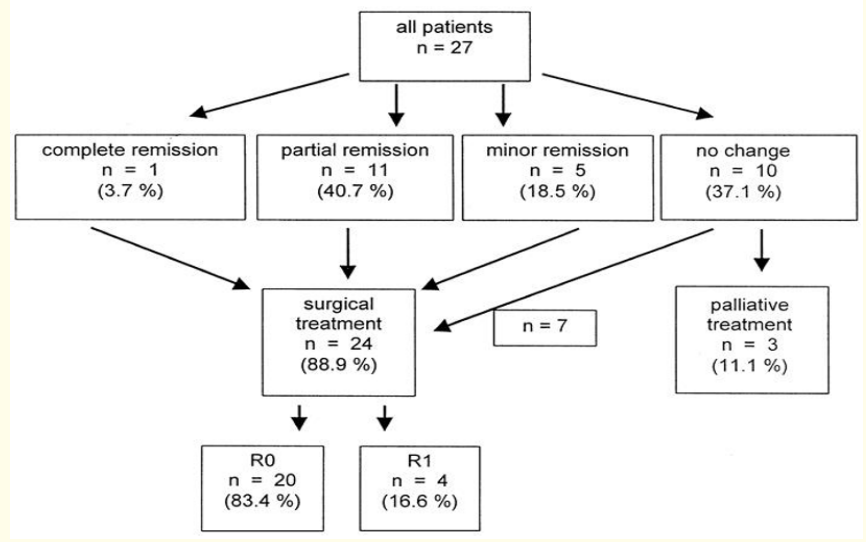

Figure 10: Schematic protocol of treatment of esophageal carcinoma should as shown [8].

Radical surgical approach was used $84,6 \%$ patients and the rest patients $15,4 \%$ had palliative surgery. The most used techniques was gastroplasty which was the best and from palliative sugery was gastrostomy. Postoperative complications were present in $23,1 \%$ of cases.

Reactions of tumor on chemotherapy and radiotherapy are different, sometimes even remission of malignant cell is possible. Even in that cases radical surgical approach remains the best method in every case after chemotherapy and radiotherapy.

\section{Bibliography}

1. Gerzić ZB. Hirurgija jednjaka. Beograd: Verzalpres (1998).

2. Morris PJ and Malt RA. Oxford texbook of surgery. New York: Oxford University Press (1994): 893-907.

3. Prpić I., et al. Kirurgija za medicinare. Zagreb: Skolska knjiga (1995): 272-277.

4. Kumar V., et al. Basic Pathology. Phyladephia: W.B. Saunders Company (1994): 482-483.

5. Štulhofer M. Kirurgija probavnog sustava. Zagreb: Medicinska Naklada (1999): 2-90.

6. Bradić I. et al. Kirurgija. Zagreb: Medicinska naklada (1995)

7. Hsieh JS., et al. "Laparoscopic Witzel gastrostomy—a reappraised technique”. Surgical Endoscopy 21.5 (2007): 793-797.

8. Guo K., et al. "The predictive value of histological tumor regression grading (TRG) for therapeutic evaluation in locally advanced esophageal carcinoma treated with neoadjuvant chemotherapy". Chinese Journal of Cancer 31.8 (2012): 399-408. 
9. Wu TT., et al. "Excellent interobserver agreement on grading the extent of residual carcinoma after preoperative chemoradiation in esophageal and esophagogastric junction carcinoma: a reliable predictor for patient outcome". The American Journal of Surgical Pathology 31.1 (2007): 58-64.

10. Iyer R., et al. "Controversies in the multimodality management of locally advanced esophageal cancer: evidence-based review of surgery alone and combined-modality therapy". Annals of Surgical Oncology 11.7 (2004): 665-673.

11. Imdahl A., et al. "Impact of neoadjuvant therapy of perioperative morbidity in patients with esophageal cancer". The American Journal of Surgery 187.1 (2004): 64-68.

12. Doty JR., et al. "Postesophagectomy morbidity, mortality, and length of hospital stay after preoperative chemoradiation therapy". The Annals of Thoracic Surgery 74.1 (2002): 227231.

13. Yano M., et al. "Correlation between histological effects on the main tumors and nodal status after chemoradiotherapy for squamous cell carcinoma of the esophagus". Journal of Surgical Oncology 89.4 (2005): 244-250.

14. Prenzel KL., et al. "Reduced incidence of nodal micrometastasis after major response to neoadjuvant chemoradiation in locally advanced esophageal cancer". Annals of Surgical Oncology 14.2 (2007): 954-959.

15. Brücher BL., et al. "Neoadjuvant therapy of esophageal squamous cell carcinoma: response evaluation by positron emission tomography". Annals of Surgery 233.3 (2001): 300-309.

16. Siewert JR and Hölscher AH. "Current strategy in surgery for esophageal cancer”. Annali Italiani Di Chirurgia 63.1 (1992): 13-18.

17. Fink U., et al. "Esophageal cancer: Neoadjuvant therapy for squamous cell esophageal carcinoma”. Annals of Oncology 5.3 (1994): S17-S26.

18. Raoul JL., et al. "Neoadjuvant chemotherapy and hyperfractionated radiotherapy with concurrent low-dose chemotherapy for squamous cell esophageal carcinoma". International Journal of Radiation Oncology Biology Physics 42.1 (1998): 29-34.

19. Sobin LH and Fleming ID. "TNM Classification of Malignant Tumors, fifth edition (1997). Union Internationale Contre le Cancer and the American Joint Committee on Cancer". Cancer 80.9 (1997): 1803-1804.
20. Mandard AM., et al. "Pathologic assessment of tumor regression after preoperative chemoradiotherapy of esophageal carcinoma. Clinicopathologic correlations". Cancer 73.11 (1994): 2680-2686.

Volume 3 Issue 11 November 2019

(c) All rights are reserved by Edina Tanović., et al. 